\title{
Optical and infrared coupled full-field measurements at a mesoscopic scale
}

\author{
by L. Bodelot* ${ }^{*}$ L. Sabatier ${ }^{*}$ E. Charkaluk* and P. Dufrénoy* \\ *Laboratoire de Mécanique de Lille - UMR CNRS 8107 - Villeneuve d'Ascq, France
}

\begin{abstract}
Mechanical fields in a metallic sample are often considered homogeneous at the sample scale. Nevertheless, metallic materials are made of an aggregate of grains. Under mechanical loading, the grains undergo different levels of deformation according to their crystallographic orientation with respect to the loading axes. The deformation is thus heterogeneous at the mesoscopic scale of grains. Furthermore, it is well-known that thermal effects accompany deformation. It can thus be assumed that temperature is also heterogeneous at this scale. This study aims at observing the heterogeneities of kinematic and thermal data at the grain scale of a polycristal under cyclic loading.
\end{abstract}

\section{Introduction}

Many isotropic metallic materials are made of an aggregate of grains of random crystallographic orientations. Among these grains, some are favourably oriented for plastic gliding with respect to the loading axes. As a result, even under a uniaxial monotonous mechanical loading, local heterogeneities appear because these grains, which are thus submitted to higher level of stress, can exhibit plastic strains whereas others still undergo a pure elastic response [1]. As plasticity triggers the appearance of slip bands at the surface of the material, the strain heterogeneities are translated into heterogeneities of relief at the sample's surface. Such heterogeneities of relief can be easily observed nowadays thanks to high resolution imagery techniques like scanning electron microscopy or atomic force microscopy [2]. Since the pioneering work of Farren and Taylor, it is well-known that plastic deformation is also accompanied by heat dissipation conducting to thermal evolutions which can be detected at the structure scale during the loading of the specimen. In their work, they used a calorimeter or thermocouples to evaluate the heat dissipated during the loading of specimens and then realized energy balances [3, 4]. Hence the information provided by these studies lead to the conclusion that, under monotonous or cyclic loading, the material behaviour is mechanically and probably thermally different from one grain to another.

Studies exploiting the thermal dissipation of metals due to plasticity have already been published. Under cyclic loading, Luong [5] and Curà [6] determined the fatigue limit of a sample thanks to the evolution of its mean temperature. This piece of information combined with a probabilistic model of fatigue also allows Doudard to predict fatigue scatter [7]. As the fatigue limit determination worked on steels but gave bad results on aluminium specimens, it was supposed that the global temperature of the sample was not a sufficient indicator for damage. Then, other research teams focused on the determination of heat sources from temperature measurements [8]. Nevertheless all these studies take place at a very macroscopic scale, since they are based on the mean temperature of the specimen, whereas thermomechanical fields in polycrystalline metals are not homogeneous. A way to account for this is to perform full-field measurements. Hence thermal full-field measurements were carried out to investigate instabilities like Portevin-Le Chatelier effect [9] and digital image correlation was used to get strain fields and study localization phenomena like necking or Lüders bands [10]. The link between thermal and kinematic behaviours has also been investigated to perform energy balances: couplings of thermal and kinematic field measurements have indeed been attempted either on both sides of a sample [11] or on separate samples [12]. Such studies provide more "local" energy balances but they remain at the scale of the sample whereas plasticity appears at the scale of grains. There is thus a lack of simultaneous information about the kinematic and thermal behaviours of the material at the mesoscopic scale of damage.

Performing thermomechanical measurements on a metallic sample at this scale could be useful, as it would give information about the behaviour of an Elementary Representative Volume of a polycristal and then could help to enhance polycrystalline models. For that purpose an original fully-coupled measurement technique was designed to get, at the same time and on the same surface, strains and thermal emissions at the mesoscopic scale of grains. The full-field measurements methods used in this work are first presented, namely Infrared Thermography (IRT) for temperature field measurements and Digital Image Correlation (DIC) for strain field measurements. Necessary constraints and precautions to reach fully-coupled recordings are specified as well as the choice of the samples' material. Then the specific use of the IRT camera to perform quantitative infrared measurements at the mesoscopic scale is developed. Finally, kinematic and thermal results obtained on AISI $316 \mathrm{~L}$ austenitic stainless steel are presented and discussed. In this communication, the presented results are obtained in the case of a cyclic loading. 


\section{Experimental setup for fully-coupled measurements at the mesoscopic scale}

\subsection{Thermal measurements at the mesoscopic scale}

The IRT camera used in this work is a Cedip Jade III. Its infrared sensitive part is a Focal Plane Array (FPA) of 320 by $240 \mathrm{InSb}$ detectors cooled down at $77 \mathrm{~K}$ by a rotary Stirling and working in the spectral range from 3 to $5 \mu \mathrm{m}$. Its maximum frame rate in full frame is $170 \mathrm{~Hz}$ and the dynamic range of digital levels is 14 bits. This camera is used with a high magnification lens known as G1 since the spatial resolution matches the size of one detector, which is 30 by $30 \mu \mathrm{m}^{2}$. This leads to a 9.6 by $7.2 \mathrm{~mm}^{2}$ observation zone but as the $\mathrm{G} 1$ lens induces optical distortions, the working zone is here narrowed to 5 by $5 \mathrm{~mm}^{2}$.

The way this camera is used to perform infrared measurements at the grain scale of a sample will be described further in paragraph 3.

\subsection{Kinematic measurements at the mesoscopic scale}

Images of the sample are grabbed during the test thanks to a CCD Camera Jai CV-M4+. This camera is made of a matrix of 1368 by 1024 detectors sensitive to visible wavelengths from 0.38 to $1 \mu \mathrm{m}$; it delivers greyscale images and the dynamic range of their digital levels is 10 bits. A TAMRON 23FM50SP $50 \mathrm{~mm}$ lens associated to extension tubes enables a high spatial resolution of 6.5 by $6.5 \mu \mathrm{m}^{2}$ per pixel leading to a working zone of approximately 8.9 by $6.7 \mathrm{~mm}^{2}$. Although no spatial distortion near to the borders is observed, the working zone is reduced to 5 by $5 \mathrm{~mm}^{2}$, in order to match the infrared data.

Displacement and strain fields are then obtained through DIC performed on the images captured during mechanical deformation of the sample. The displacement field of one image is determined with respect to a reference image thanks to the DIC software Correli ${ }^{\text {LMT }}$ developed at LMT laboratory (Cachan, France). The principle is the following. The reference two-dimensional image is divided into square regions (called Zone of Interest, ZOI); then, the DIC software matches these ZOI between one image at a time $t_{i}$ and the reference image at a time $t_{0}$, thanks to an algorithm based on Fast Fourier Transformation [13]. The results of the DIC analysis are then two-dimensional displacement and strain fields for each time $t_{i}$. The principle of correlation is based on pattern recognition. The pattern has to be randomly obtained and has to cover a wide range over the greyscale. In some particular cases, such a random pattern appears naturally on the tested material so that correlation can be performed on images of the raw material. For example, DIC was recently computed directly from images of the metallic phases of polished and electrochemically etched duplex stainless steel [14]. In more general cases, the pattern has to be created artificially: the sample is then usually covered with black and white paint in order to obtain a random speckle. In the present case, a coating has to be applied since the sample is polished as a mirror and the working scale is not as fine as the one of El Bartali.

\subsection{Principle of the coupling}

In order to get temperature and strain fields at the same time, a sample can be observed on one side with a CCD camera, in order to perform DIC, and on the other side with an IRT camera, in order to determine its temperature [15]. To process such data, it must be assumed that the kinematic and thermal responses are the same on each face of the sample. However, the objective of the present work is to get these data at the grain scale and to establish a link between them; thus the former method is not suitable since the microstructure is different from one face to the other. Therefore, it is necessary to observe the same area of the sample simultaneously with both IRT and CCD cameras: this is the objective of the original coupling method developed in this study.

In order to realize this simultaneous observation, a "filter-mirror" is placed in front of the sample with an angle of $45^{\circ}$ with respect to the sample's surface. On the one hand, this filter-mirror behaves like a filter since it only lets the infrared radiation go towards the IRT camera placed ahead of the sample. On the other hand, it also behaves like a mirror since it reflects the rest of the radiation, among which the visible radiation, in the direction of the CCD camera (figure 1).

The "filter-mirror" allows a simultaneous observation of the same zone and high magnification lenses give access to high spatial resolution images. However, reaching fully-coupled measurements supposes that the coating applied on the sample meet conditions for both DIC and IRT measurements. As a matter of fact, DIC has to be performed on optical images of random and contrasted aspect, covering a large range of grey levels, whereas infrared measurements require to be performed on high emissivity objects, which can be obtained thanks to a dark and homogeneous coating. As a result, the coating must be a speckle when observed by the CCD camera as well as dark and homogeneous when observed by the IRT camera. In consequence, a special coating has been developed; it is suitable, at the working scale, for both techniques and thus permits of fullycoupled measurements. The coating seen by both cameras equipped with the lenses described above is presented on figure 2 . 

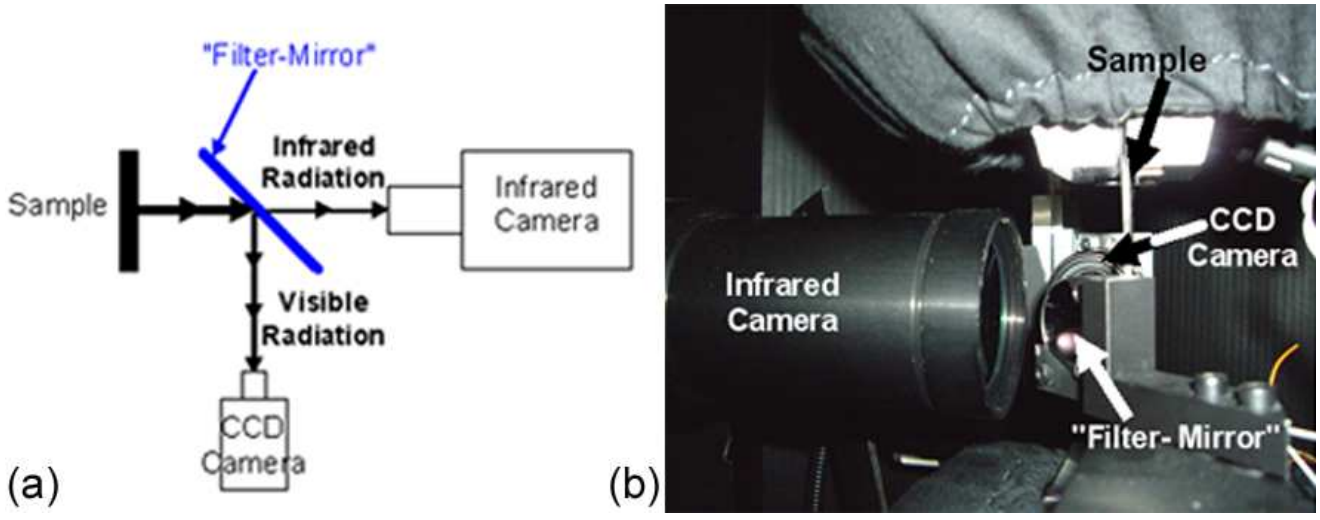

Fig. 1. Principle of the coupling method (a) and view of the experimental setup (b)

(a)

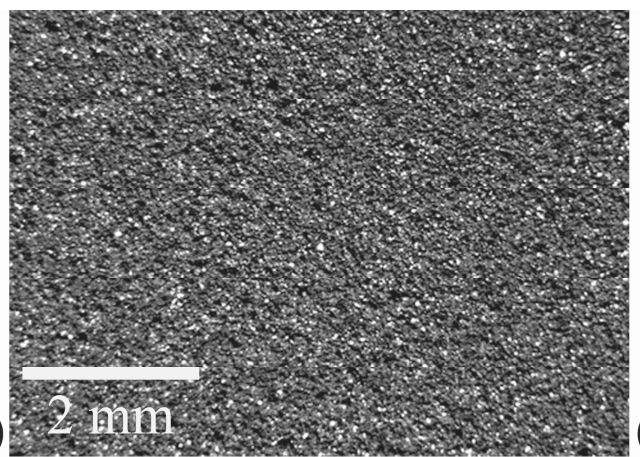

(b)

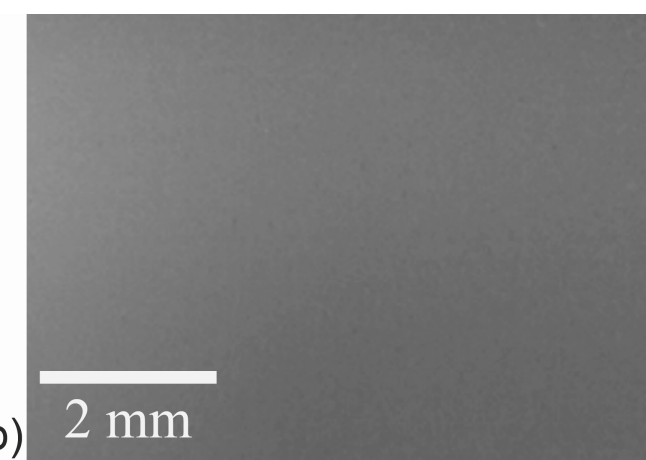

Fig. 2. Specially designed coating observed by the CCD camera equipped with the $50 \mathrm{~mm}$ lens and extension tubes (a) and by the IRT camera equipped with a G1 lens (b)

\subsection{Samples material}

The choice of the material is also a crucial issue. First of all, the material's thermal diffusivity (ratio of thermal conductivity to volumetric heat capacity) must be as low as possible so that heat generation is not dissipated nor homogenised too quickly with respect to the frame frequency of the IRT camera. This is actually essential to be able to capture the thermal field heterogeneities. Secondly, single phase steel is preferred in order that the thermal properties can be considered identical in all grains. Finally, the grain size has to be large enough so that several pixels of both IRT and CCD matrixes can stare at one grain. This last condition leads us to use a metal having a mean grain size of roughly $100 \mu \mathrm{m}$.

In order to respect as well as possible all the above-mentioned constraints, the chosen material is an AISI $316 \mathrm{~L}$ austenitic stainless steel since it meets the diffusivity's criterion (table 1) and is a single phase steel. Its initial grain size is of about $10 \mu \mathrm{m}$, which is not enough. However, in the case of AISI $316 \mathrm{~L}$, this size can easily be raised up to about $130 \mu \mathrm{m}$ (considering twins) thanks to a heat treatment at $1200{ }^{\circ} \mathrm{C}$ for $2 \mathrm{~h}$, immediatel y followed by a water quench. Samples of the material before and after the heat treatment are chemically etched and examined under a microscope to underscore the grain growth (figure 3 ).

Table 1. Thermal properties for several metals at $20{ }^{\circ}[16,17]$

\begin{tabular}{|c|c|c|c|c|}
\hline Metal & $\begin{array}{c}\text { Density } \rho \\
\left(\mathrm{kg} \cdot \mathrm{m}^{-3}\right)\end{array}$ & $\begin{array}{c}\text { Specific Heat } \mathrm{c}_{\mathrm{p}} \\
\left(\mathrm{J} \cdot \mathrm{kg}^{-1} . \mathrm{C}^{-1}\right)\end{array}$ & $\begin{array}{c}\text { Conductivity } \mathrm{k} \\
\left(\mathrm{W} \cdot \mathrm{m}^{-1} . \mathrm{C}^{-1}\right)\end{array}$ & $\begin{array}{c}\text { Diffusivity } \mathrm{\alpha} \times 10^{5} \\
\left(\mathrm{~m}^{2} . \mathrm{C}^{-1}\right)\end{array}$ \\
\hline Copper & 8,940 & 380 & 389 & 11.4 \\
\hline Aluminium & 2,700 & 860 & 200 & 8.6 \\
\hline Brass & 8,500 & 370 & 100 & 3.3 \\
\hline Steel $(0.1 \% \mathrm{C})$ & 7,850 & 490 & 46 & 1.2 \\
\hline AISI 316L Steel & 8,000 & 500 & 15 & 0.4 \\
\hline
\end{tabular}


(a)
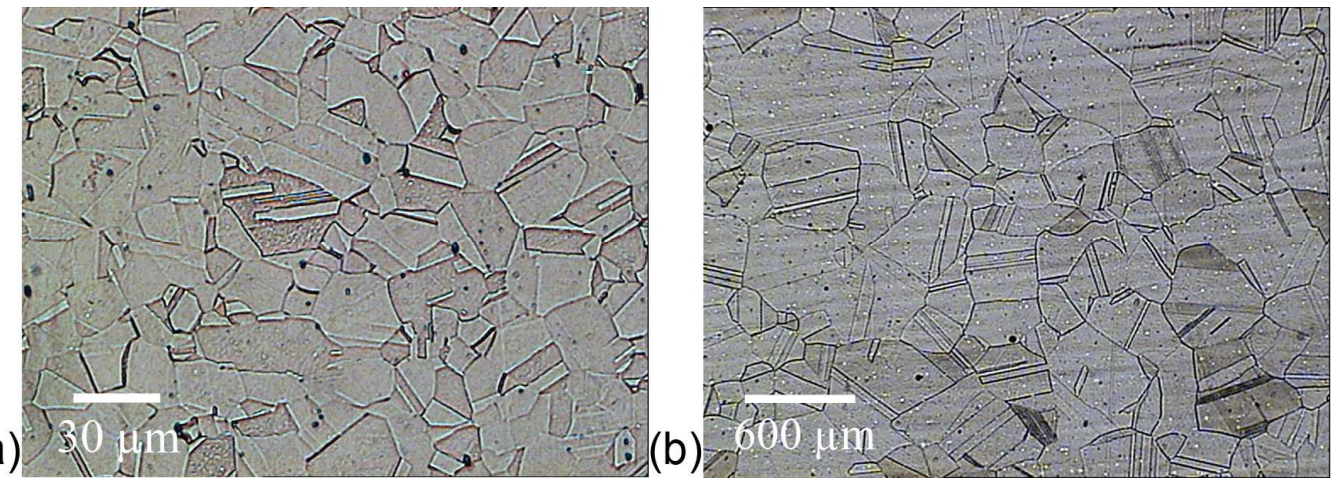

Fig. 3. Initial microstructure of AISI $316 \mathrm{~L}$ (a) and microstructure obtained after heat treatment: $1200 \mathrm{C}$ for $2 h$ and water quench (b)

\section{Quantitative infrared measurements}

To perform accurate quantitative infrared thermography, a precise calibration must be performed, the emissivity of the object has to be determined and some precautions must be taken.

\subsection{Calibration law}

The calibration law gives a link between temperatures and thermal emissions. Nevertheless in FPA cameras, each detector has its own behaviour which is even different each time the camera is switched on. Thus, in standard use, the response of each detector is corrected by a gain and an offset in order to make it as close as possible to the mean response of the matrix, this is the Non Uniformity Correction (NUC) procedure. If despite such a correction a detector's response is still too far from the mean value, it is considered as "bad" and its response is replaced by the one of its first "good" neighbouring detector or by an average made over several "good" neighbouring detectors, this is the Bad Pixel Replacement (BPR) procedure. These NUC and BPR procedures tend to make the response of the detectors of the matrix uniform; as a result a unique calibration law for the whole matrix of detectors can be established by exposing the detector array of the camera in front of an extended blackbody at different controlled temperatures. In many applications, cameras are used this way but the fact that a detector's response can be replaced by another will raise problems if one expects to observe heterogeneities at a small scale, like in our case, or to calculate gradients or Laplacians over several thermal images [18].

Therefore, in this work, the standard procedure including NUC and BPR is bypassed and the calibration law is established detector by detector. This implies that the unique calibration law is replaced by a set of 76,800 calibration laws. The procedure is the following. The matrix of detectors is exposed in front of an extended blackbody at a number $N$ of stabilized and controlled temperatures along the working temperature range. This provides $N$ relationships between a detector's signal, given in Digital Level $\left(D L_{n}\right)$, and a known temperature of the blackbody $T b_{n}$. For each detector, the calibration law is a polynomial function fitting the $N$ previous relationships, obtained thanks to the calibration procedure:

$$
T b_{n}=\sum_{i=0}^{5} c_{i} D L_{n}^{i}
$$

The coefficients $c_{i}$ are determined by a least squares approximation. The degree of the polynomial is chosen equal to 5 , which is sufficient to describe the detectors' responses. Once the calibration laws are computed, the response of each detector is tested: if the difference between its response and the mean response is above a given threshold $(80 \mathrm{mK})$, its position is identified. Generally, $0.5 \%$ of "bad" pixels are listed over the whole matrix. As most of the "bad" pixels are located on the borders, results are thus better when a central zone is considered. The calibration curves obtained for all detectors are plotted on figure 4 in order to emphasize the heterogeneity of their response. At the top and at the bottom, two curves can be made out; they correspond to defective detectors.

As the calibration is influenced by many parameters (distance to the object, lens used, working temperature, integration time, frame rate ...), it is necessary to do a new calibration for each test configuration [19] and each time the camera is switched on. Moreover, in our particular case, the "filter-mirror" which stands between the IRT camera and the sample has a transmission curve depending on the wavelength. As a result, the transmission of the infrared radiation is not perfectly complete and this has to be taken into account during the calibration procedure. This implies that during the calibration, the "filter-mirror" has to be placed in front of the blackbody, exactly in the same configuration than for the tests. 


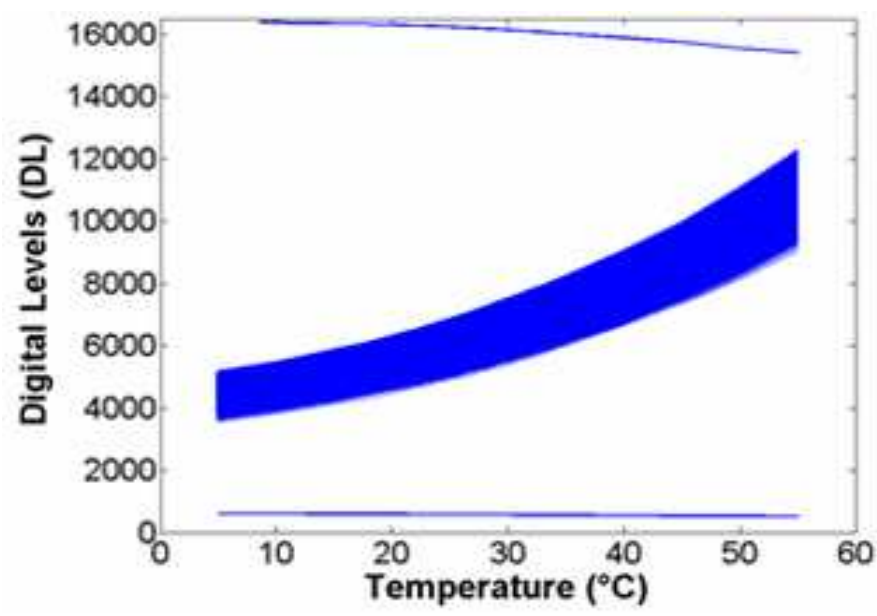

Fig. 4. Calibration curves obtained for each of the 76,800 detectors during a calibration procedure. The outstanding curves at top and bottom correspond to defective detectors

\subsection{Emissivity determination}

The ideal object for IRT measurements is the blackbody, whose emissivity is equal to 1. In this particular case, the infrared radiation received by the camera is only due to the object's temperature since no reflection from the environment is transmitted (it is actually completely absorbed). As a consequence, IRT measurements have to be performed on high emissivity objects. This is usually obtained by applying a dark and homogeneous coating over the object. Nevertheless, even very high quality coatings like high emissivity black paint, have an emissivity of 0.98 , which is still lower than the one of a blackbody [20]. As the calibration law has been established thanks to a blackbody, the emissivity of the object (or its coating) must be known in order to take reflections from the environment into account when thermal emissions are converted into temperatures.

The emissivity determination can be realized through a classically used method which consists in a comparison between the radiation of the object and the one of an extended blackbody, both being at the same temperature [20]. Most of the time, the sample's temperature is measured thanks to a thermocouple. However the thermocouple placed at the rear side of the sample creates a heat sink which, at our working scale, highly disturbs the thermal measurements, leading to non exploitable data. As a consequence, another way to carry out the emissivity determination has to be considered. In this second method, the IRT camera and an extended blackbody are placed symmetrically in front of the sample, each device making an angle smaller than $45^{\circ}$ with the normal to the sample's surface. Then, the blackbody is given two temperatures $T b_{1}$ and $T b_{2}$ corresponding to two temperature responses $T s_{1}$ and $T s_{2}$ of the sample, which reflect a part of the blackbody radiation. Finally the emissivity $\varepsilon$ will be given by the following relationship:

$$
\varepsilon=1-\frac{T s_{2}-T s_{1}}{T b_{2}-T b_{1}}
$$

As the experimental setup to perform this emissivity determination is under progress, the emissivity will be considered equal to 1 for the temperature measurements presented in this paper.

\subsection{Precautions}

IRT cameras are submitted to thermal drift. This means that, even if the camera observes a stable thermal scene (for example, a blackbody at a given controlled temperature), the result will be different if the measurement is made just after the camera has been switched on or after several hours: the temperature "seen" by the camera will be a few degrees higher. To avoid facing with this phenomenon, the IRT camera is turned on more than 4 hours before beginning any measurements including calibration. As the emissivity of our coating is necessary lower than 1, the sample is likely to reflect environment radiations. The working place around the camera is thus prevented from reflections from the environment thanks to black tissue and every element close to the camera is also covered by black tissue or painted with high emissivity black paint to avoid any residual reflection. Moreover, to reduce the influence of the environment as much as possible, light is turned off and the room temperature is kept steady thanks to air conditioned. 


\section{Experiments}

\subsection{Samples}

$3 \mathrm{~mm}$ thick specimens are cut out by electroerosion from a thick laminated sheet of $316 \mathrm{~L}$ and are heat treated as described in 2.4. After the heat treatment at high temperature, samples are covered by a carbon layer due to oxidation under air. This layer is removed during the polishing since samples are mechanically polished up to $1 \mu \mathrm{m}$ on faces and up to grit $4000 \mathrm{SiC}$ paper on the edges. Final specimens are $2 \mathrm{~mm}$ thick plates having a gage area of 10 by $10 \mathrm{~mm}^{2}$ (figure 5 ).

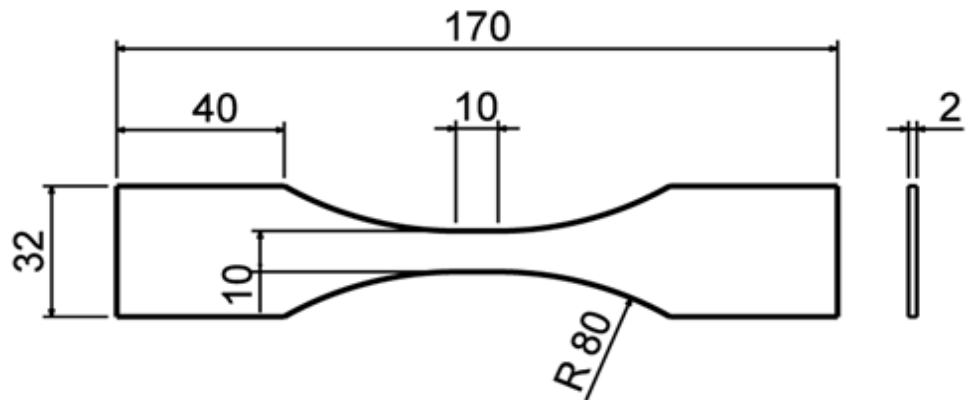

Fig. 5. Plan of the final samples

\section{$4.2 \quad$ Tests}

Experiments are performed on an electro-mechanical Instron 4508 machine which enables tests up to $300 \mathrm{kN}$ on a speed range from 0.1 to $100 \mathrm{~mm} / \mathrm{min}$. During the test, displacement, strain and temperature fields are recorded in the middle of the gage zone thanks to the fully-coupled measurement technique presented in the previous paragraphs. The load applied to the sample is also recorded during the tests.

The test carried out in this work is a $2.8 \mathrm{~Hz}$ cyclic test at a ratio $R_{\sigma}$ of 0.1 and a mean stress of $85 \mathrm{MPa}$. The stresses due to the cyclic loading applied on the sample during the test are plotted on figure 6 .

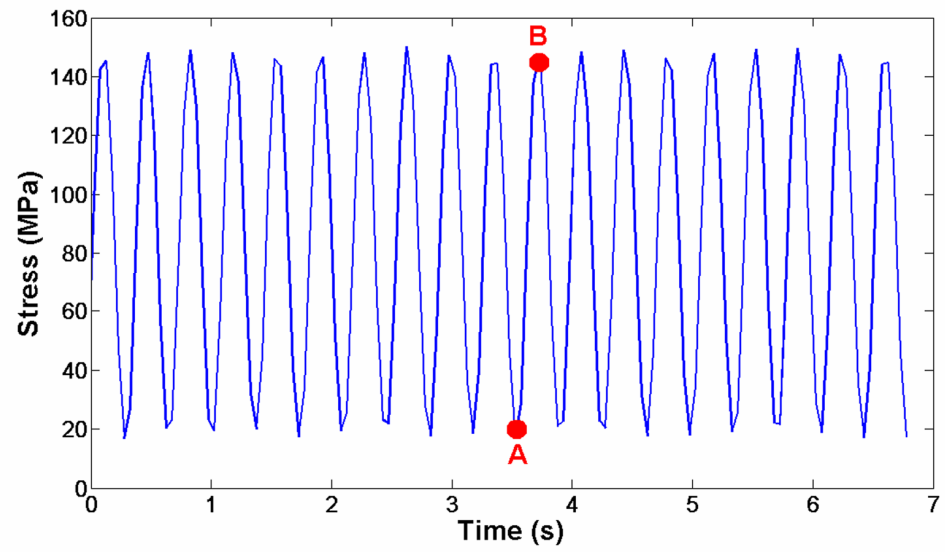

Fig. 6. Stress cycles versus time applied to the sample. Points $A$ and $B$ correspond to the instants when the strain fields are studied

\section{Results and discussion}

\subsection{Strain measurements}

Strain fields corresponding to the instants marked A and B on figure 6 are computed by DIC with respect to the reference image of the unloaded sample. The working zone is a 5 by $5 \mathrm{~mm}^{2}$ square located in the middle of the sample's gage area. Here, DIC is performed on ZOIs of 16 by 16 pixels; this leads to a spatial resolution of 104 by $104 \mu^{2}$ as far as strain data are concerned. The scale of figure 7 is the same for both fields and strain values greater than $0.2 \%$ are represented in white. 


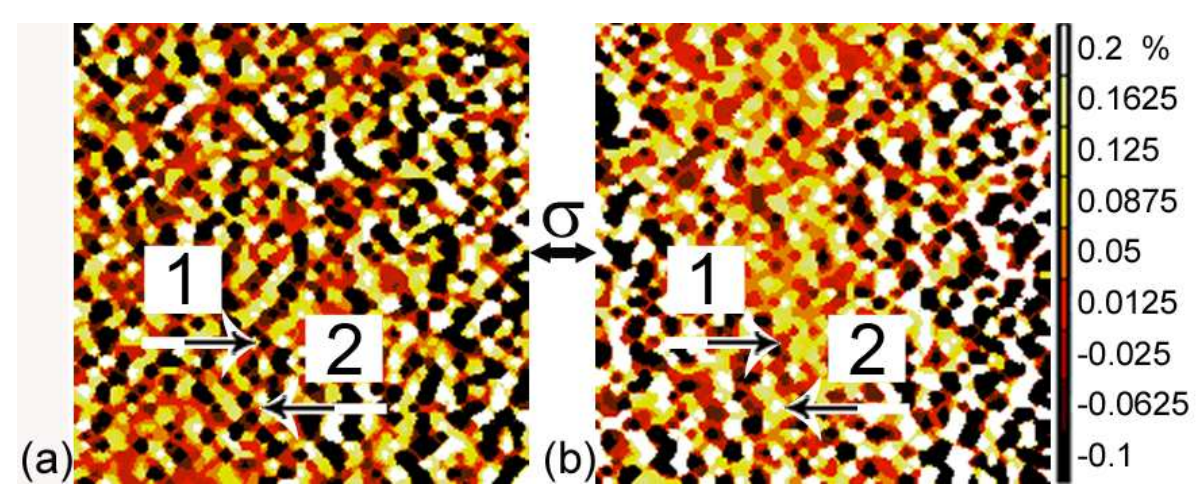

Fig. 7. Strain fields obtained by DIC at instants $A(a)$ and $B(b)$ of figure 6 , over a 5 by $5 \mathrm{~mm}^{2}$ zone within the gage area. The scale is related to both fields and points 1 and 2 exhibit different levels of strain.

Negative values appear on both fields of figure 7. The sample is actually submitted to a uniform uniaxial tensile load but the grains have different crystallographic orientations; thus the effective stress to which they are really submitted depends on their orientation with respect to the loading axe and is also influenced by the neighbouring grains. As a result some grains can be under compression during a tensile load.

Figure 7a corresponds to an instant when the sample is submitted to the lowest stress whereas figure $7 \mathrm{~b}$ is related to the highest stress. As a result, the level of deformation is lower on figure $7 \mathrm{a}$ than on figure $7 \mathrm{~b}$ : shades are darker on figure $7 \mathrm{a}$. The cumulated mean axial strain value over the studied zone is indeed $0.024 \%$ for figure $7 \mathrm{a}$ and $0.061 \%$ for figure $7 \mathrm{~b}$.

Both fields exhibit heterogeneities since the strains are within a range from $-0.1 \%$ to $0.2 \%$. Points 1 and 2 marked on figure 7 are examples of this phenomenon. On the field of figure $7 \mathrm{~b}$, point 1 is submitted to a low level of strain (dark shade) whereas point 2 exhibits a higher strain (light shade). The strain level is indeed $0.0026 \%$, at point 1 and $2.8 \%$ at point 2 . Considering the resolution of the strain fields, these two points are definitely in different grains. As a result these strain fields account for a different kinematic behaviour from one grain to another: some grains are certainly still under an elastic state whereas others already exhibit plastic strains as it was shown by Korsunsky through neutron diffraction measurements realized at the grain scale of a stainless steel under cyclic loading [1].

\subsection{Thermal measurements}

The heterogeneities of thermal fields captured at instants $A$ and $B$ can not be related to the strain fields when observed at the naked eye. As a consequence, points 1 and 2 exhibiting very different values of strain on figure 7 are analysed and the time-filtered thermal evolutions versus time of the corresponding infrared detectors are plotted on figure 8.

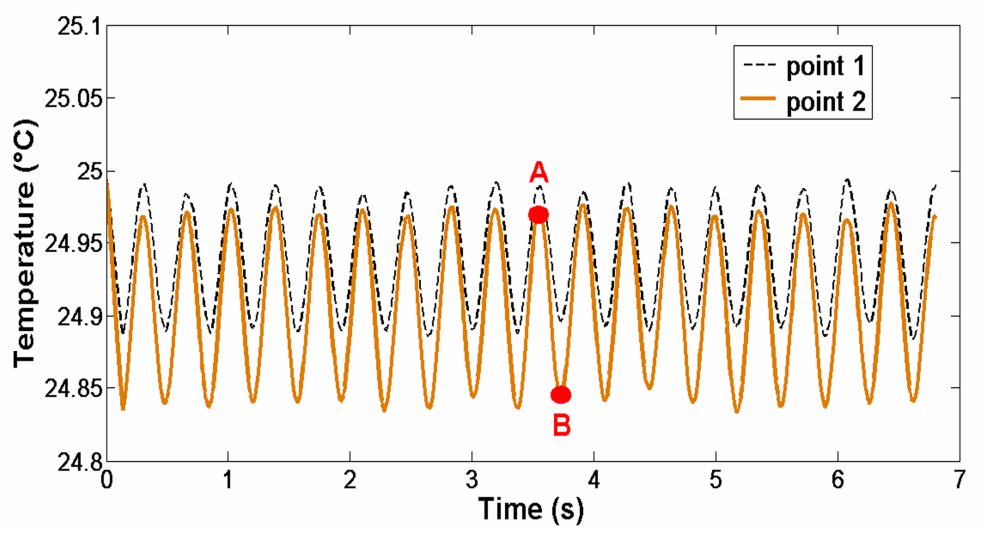

Fig. 8. Thermal evolutions versus time of points 1 and 2 of Figure 7. Points $A$ and $B$ correspond to the instants of the studied strain fields.

The thermal evolutions of points 1 and 2 follow the same trend but with different levels. Lets us remind that the thermoelastic coupling effect leads to a temperature raise of a sample submitted to an elastic compression load and to a temperature drop under elastic tensile load. This explains why, at instant $A$, when the stress is minimal, the temperatures of points 1 and 2 reach their maximum value and why, inversely at instant $B$ when the stress is maximal, the temperatures of points 1 and 2 reach their minimum value. There is thus a phase opposition between stress and thermal signals of figures 6 and 8 . 


\subsection{Link between kinematic and thermal data}

As already mentioned points 1 and 2 pinpointed out on figure 7 correspond to different grains and are not submitted to the same levels of strain. Figure 8 shows that their thermal behaviours are different as well. As point 2 exhibits a higher strain than point 1 , its thermal decrease is more important than the one of point 1 from the beginning of the cycling.

It has been established [8] that the temperature variation $\Delta \theta_{\text {the }}$ caused by the thermoelastic coupling effect on a sample submitted to a cyclic loading, whose amplitude is $\Delta \sigma$ at a frequency higher than $1 \mathrm{~Hz}$, is well approximated by:

$$
\Delta \theta_{\text {the }} \approx \frac{\alpha T_{0} \Delta \sigma}{\rho C}
$$

In this relationship, $\alpha$ denotes the isotropic thermal dilatation, $\rho$ the density of the material and $T_{0}$ the initial temperature. Strain fields of figure 7 show that point 2 is submitted to higher strain and thus stress amplitudes than point 1 . On figure 8 , the temperature amplitude at point 2 is higher than the one at point 1 . These results are thus in good agreement with relationship (3).

Nonetheless with a strain value of $2.8 \%$, point 2 undergoes plasticity and it is known that plasticity entails a thermal dissipation. However the temperature evolution of point 2 looks purely sinusoidal and thus seems to be only linked to the thermoelastic coupling effect. No increase of the temperature mean value can clearly be noticed. This means that the thermal increase due to plasticity, which is of second order compared to the thermoelastic effect, was not captured during these few cycles. This thermal dissipation signal has certainly been diffused within the sample. Accordingly, it may be necessary to analyse thermal sources, instead of studying solely the temperature.

\section{Conclusion}

In this study and for the first time, fully-coupled kinematic and thermal measurements have been performed on austenitic stainless steel at the grain scale. The original setup developed shows that it is possible to highlight heterogeneities at this scale for both kinematic and thermal behaviours. The kinematic and thermal data observed in this test are thus typical from the heterogeneous deformation mechanisms which take place in a polycristal under cyclic loading and which have been described in the introduction. However temperature data seem to be significantly affected by diffusion and this last observation tends to confirm that temperature may not be used as a damage indicator and that processing thermal data to get thermal sources could thus be more promising.

Nevertheless, these results show that it is possible to get some thermomechanical data of a Representative Volume Element of a polycristal. If the thermal fields are correctly processed within a thermomechanical framework [18], they will give access to thermal sources and since strain fields of the same zone are also available, local energy balances can be realized at the grain scale. Moreover, such information could also be used as a comparison point for results of numerical simulations developed in our laboratory like crystalline plasticity models or simulations taking into account both thermoelasticity and thermoplasticity coupling. Finally, the objective would be to enhance local non linear constitutive laws in fatigue.

\section{REFERENCES}

[1] A. M. Korsunsky, K. E. James and M. R. Daymond. Intergranular stresses in polycrystalline fatigue: diffraction measurement and self-consistent modelling, Engineering Fracture Mechanics, 71 (2004) 805-812.

[2] P. Villechaise, L. Sabatier and J. C. Girard. On slip band features and crack initiation in fatigued 316L austenitic stainless steel: Part 1: Analysis by electron back-scattered diffraction and atomic force microscopy, Materials Science and Engineering A, 323 (2002) 377-385.

[3] W. S. Farren and G. I. Taylor. The Heat Developped during Plastic Extension of Metal, Proceedings of the Royal Society, A (1925) 422-451.

[4] G. I. Taylor and H. Quinney. The latent energy remaining in a metal after cold working, Proceedings of the Royal Society, A (1933) 307-326.

[5] M. P. Luong. Fatigue limit evaluation of metals using an infrared thermographic technique, Mechanics of Materials, 28 (1998) 155-163.

[6] F. Curà, G. Curti and R. Sesana. A new iteration method for the thermographic determination of fatigue limit in steels, International Journal of Fatigue, 27 (2005) 453-459.

[7] C. Doudard, S. Calloch, P. Cugy, A. Galtier and F. Hild. A probabilistic two-scale model for high-cycle fatigue life predictions, Fatigue and Fracture of Engineering Materials and Structures, 28 (2005) 279-288.

[8] T. Boulanger, A. Chrysochoos, C. Mabru and A. Galtier. Calorimetric analysis of dissipative and thermoelastic effects associated with the fatigue behavior of steels, International Journal of Fatigue, 26 (2004) 221-229.

[9] H. Ait-Amokhtar, C. Fressengeas and S. Boudrahem. The dynamics of Portevin-Le Chatelier bands in an AlMg alloy from infrared thermography, Materials Science and Engineering, A In Press, Corrected Proof (2007). 
[10] B. Wattrisse, A. Chrysochoos, J.-M. Muracciole and M. Nemoz-Gaillard. Kinematic manifestations of localisation phenomena in steels by digital image correlation, European Journal of Mechanics, A/Solids 20 (2001) 189-211.

[11] P. R. Guduru, A. J. Rosakis and G. Ravichandran. Dynamic shear bands: an investigation using high speed optical and infrared diagnostics, Mechanics of Materials, 33 (2001) 371-402.

[12] H. Louche and A. Chrysochoos. Thermal and dissipative effects accompanying Lüders band propagation, Materials Science and Engineering, A307 (2001) 15-22.

[13] F. Hild. Multiscale displacement field measurements of compressed mineral-wool samples by digital image correlation, Applied optics, 41 (2002) 6815-6828.

[14] A. El Bartali, V. Aubin and S. Degallaix. Fatigue damage analysis in a duplex stainless steel by digital image correlation technique, Fatigue and Fracture of Engineering Materials and Structures, 31 (2007) 137-151.

[15] A. Chrysochoos, J. M. Muracciole and B. Watrisse. Experimental analysis of strain and damage localization. Continuous Damage and Fracture, Continuous Damage and Fracture, (2000) 41-51.

[16] J. F. Sacadura. Initiation aux transferts thermiques, (1982).

[17] OTUA, Propriétés physiques, in: Office Technique pour l'Utilisation de l'Acier, 2008.

[18] B. Berthel, B. Wattrisse, A. Chrysochoos and A. Galtier. Thermographic Analysis of Fatigue Dissipation Properties of Steel Sheets, Strain, 43 (2007) 273-279.

[19] H. Pron and C. Bissieux. Focal plane array infrared cameras as research tools, QIRT Journal, 1 (2004) 229240.

[20] G. Gaussorgues and S. Chomet. Infrared Thermography, London (1994). 
http://dx.doi.org/10.21611/qirt.2008.10_02_07 